\title{
Biases in Shortwave Column Absorption in the Presence of Fractal Clouds
}

\author{
W. Wiscombe, A. Marshak, A. Davis and R. Cahalan \\ NASA/Goddard Space Flight Center \\ Greenbelt, Maryland
}

\section{Introduction}

The goal of this study is to understand inter-droplet cloud absorption in horizontally inhomogeneous clouds. In order to minimize the absorption by cloud drops, a spectral band around $0.94 \mu \mathrm{m}$ with strong molecular and very weak liquid absorption has been chosen for detailed analysis. The 0.94 micron band has insignificant Rayleigh scattering and a large range of transmission (Figure 1). In other words, we have a horizontally homogeneous absorber and a horizontally inhomogeneous scatterer.

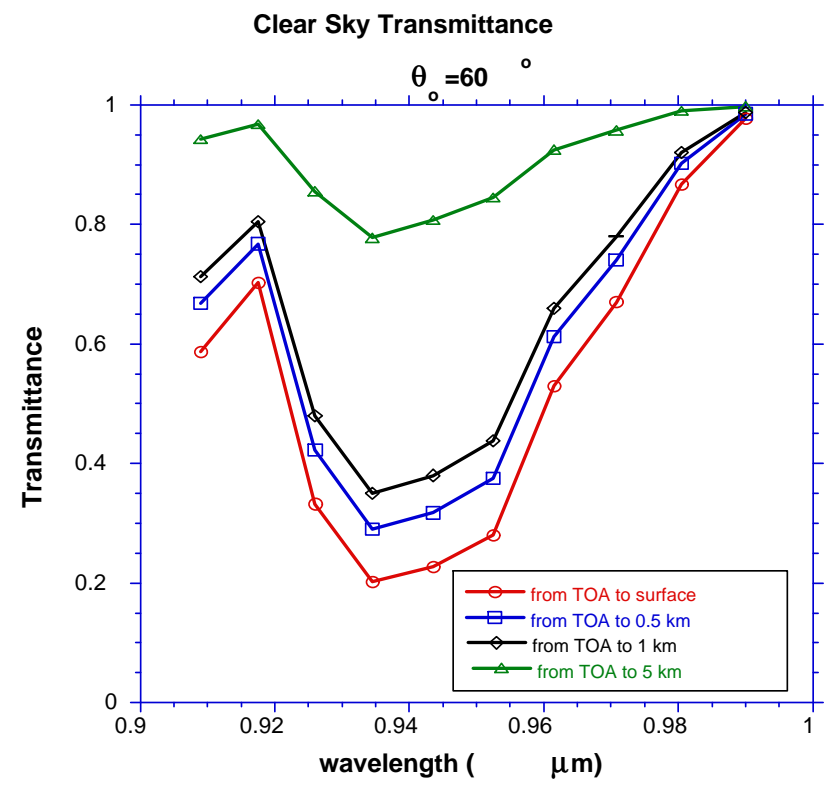

Figure 1. Water vapor transmission spectra for solar zenith angle $\theta_{0}=60^{\circ}$. From the top: from TOA to $5 \mathrm{~km}$, from TOA to $1 \mathrm{~km}$, from TOA to $0.5 \mathrm{~km}$ and, finally, from TOA to surface.

The main points to be discussed are as follows:

- a change in column absorption due to redistribution of cloud liquid water
- a change in column absorption due to horizontal fluxes

- the effects of optical and geometrical shadowings on cloud absorption

- the effects of cloud fraction and solar zenith angles.

\section{Cloud Model}

The only source of horizontal variability in our atmospheric model is clouds. To simulate the horizontal distribution of cloud optical depth, $\tau$, we use a simple three-parameter fractal model called a "bounded cascade" (Cahalan et al. 1994) for which

$$
0<\tau_{\min }{ }^{2} \tau^{2} \tau_{\max }<\langle\tau\rangle \exp \left(\frac{1-2 \mathrm{p}}{1-2^{-\mathrm{H}}}\right)<\infty
$$

where $\langle\bullet\rangle$ indicates domain-average. Parameter $\mathrm{p}$ controls the variance-to-mean ratio of cloud liquid water, while $\mathrm{H}$ determines its scaling behavior. In this study, the cascade parameters are fixed at $\langle\tau\rangle=13, \mathrm{p}=0.35$ and $\mathrm{H}=0.38$. According to the analyses of Cahalan and Snider (1989), these values provide the best fit to the diurnal average liquid water path (LWP) distribution in California marine stratocumulus (Sc).

We will distinguish between two models of the horizontal distribution of cloud optical depth "flat fractal" and "bumpy fractal" depending on which of the two parameters, cloud geometrical thickness $h$ and extinction $\sigma$, is set constant. For flat fractals, we assume $\mathrm{h}$ is constant and that both cloud top and cloud base are horizontal planes. Thus, the extinction coefficient $\sigma$ is proportional to $\tau$ (we assume it has no vertical variation). For bumpy fractals, we keep $\sigma$ constant. As a result, geometrical thickness $\mathrm{h}$ is proportional to $\tau$ with $\langle\mathrm{h}\rangle=$ $300 \mathrm{~m}$. For simplicity, we keep cloud base fixed and vary only cloud top. Examples of the two contrasting models are shown in Figure 2. 

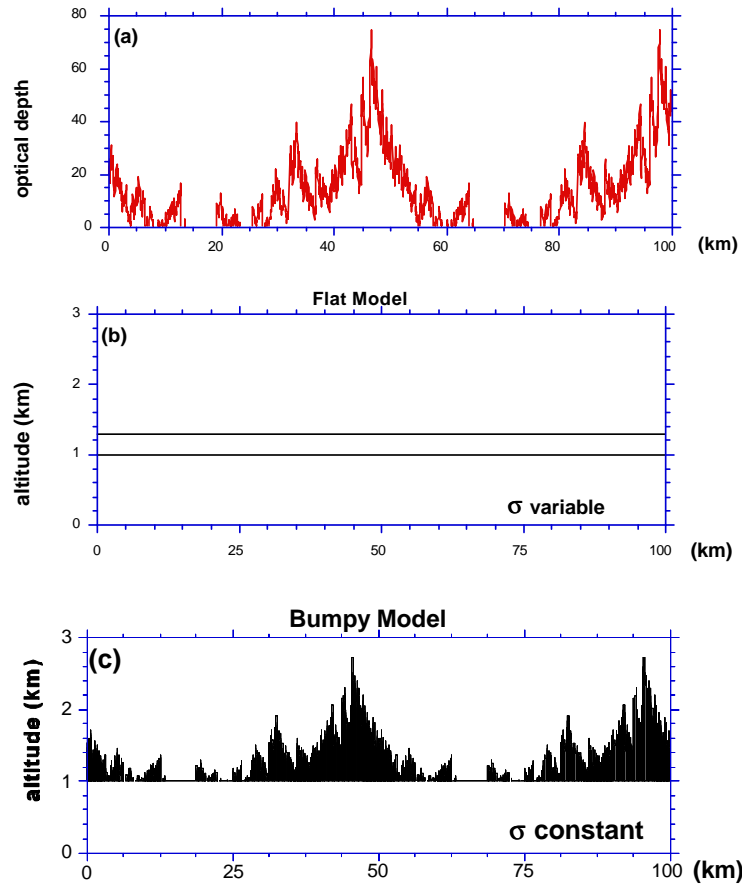

Figure 2: Fractional cloudiness $\mathrm{N}=0.777$. (a) Horizontal distribution of optical depth, the same for both models, (b) Horizontal distribution of cloud height for optical model. Constant cloud top and cloud base; thus $\sigma=\tau / h$ is variable, (c) Horizontal distribution of cloud top for geometrical model. Having $\sigma$ constant, $h_{\text {top }}=h_{\text {base }}$ $+\tau / \sigma$. To emphasize periodical boundary conditions, two basic cells of $\approx 50 \mathrm{~km}$ are plotted next to each other.

Finally, in addition to cloud inner structure simulated with the bounded cascade model, we supplement the cloud structure with gaps. For simplicity, and lacking any good theory on cloud gappiness, we subtract a constant value a $>\tau_{\min }$ from the bounded-model optical depth curve $\tau_{\text {unbroken }}(\mathrm{x})$ and set negative values to zero. This, however, does not conserve liquid water; so in order to restore the liquid water to its former value, we multiply the resulting optical depth curve by another constant, $\mathrm{b}>1$. As a result, we have

$$
\tau_{\text {broken }}(\mathrm{x})=\mathrm{b} \max \left\{\tau_{\text {unbroken }}(\mathrm{x})-\mathrm{a}, 0\right\}
$$

Constants a and $\mathrm{b}$ can be uniquely derived from the desired fractional cloudiness $\mathrm{N}$ and three parameters of bounded model: $\langle\tau\rangle, \mathrm{p}$ and $\mathrm{H}$. Figure 2 gives an example for both types of cloud model with fractional cloudiness $\mathrm{N}=0.777$, corresponding to $\mathrm{a} \approx 7.9$ and $\mathrm{b} \approx 2.4$.

\section{Cloud-Fraction Effect}

In this section we compare results for different cloud fraction $\mathrm{N}$; the same total liquid water is used in all cases. With more gaps, redistribution of cloud liquid water increases optical depth of cloudy sky.

Figure 3 shows the domain-averaged up- and downwelling fluxes together with the column absorption for different cloud fractions computed using all three radiative transfer

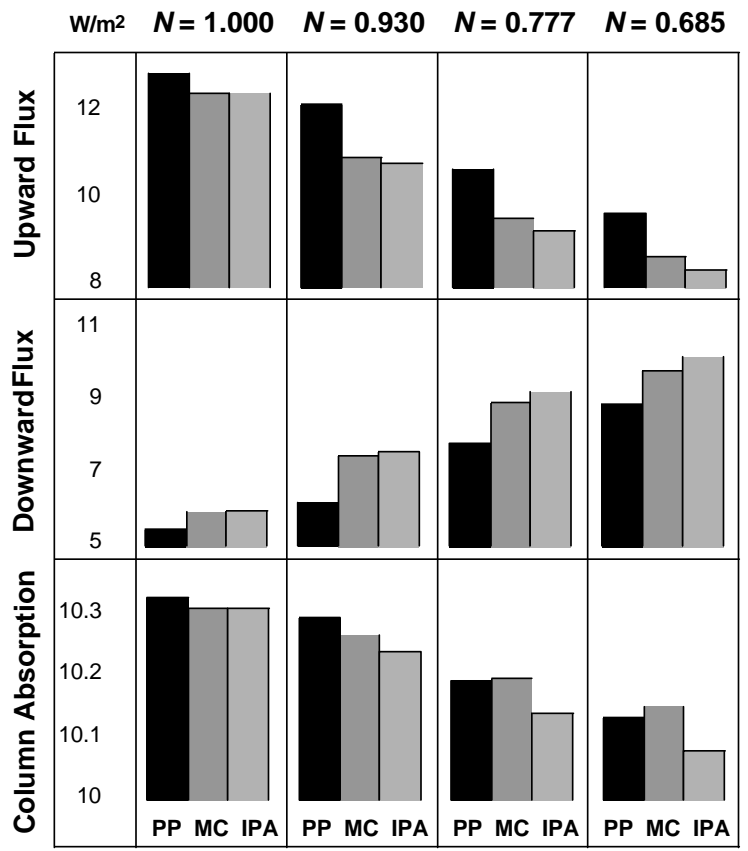

Figure 3. Domain-averaged upward flux, downward flux and resulting column absorption are plotted for four cloud fractions and three radiative transfer models: PP, MC and IPA. Flat cloud model, solar zenith angle $\theta_{0}=60^{\circ}$.

tools: plane-parallel (PP), independent pixel approximation (IPA) and Monte Carlo (MC). Obviously, with decrease of cloud fraction, reflectance decreases while transmittance increases for all three models. The decrease in absorption is also easy to understand in the framework of the IPA, because in this spectral region clear sky pixels absorb less than their cloudy counterparts. What is less obvious is that MC results for reflectance and transmittance are between PP and IPA results for any cloud fraction $\mathrm{N}$. In other words,

$$
\mathrm{R}_{\mathrm{IPA}}<\mathrm{R}_{\mathrm{MC}}<\mathrm{R}_{\mathrm{PP}}
$$




$$
\mathrm{T}_{\mathrm{IPA}}<\mathrm{T}_{\mathrm{MC}}<\mathrm{T}_{\mathrm{PP}}
$$

The difference between IPA and MC-the so-called IPAbias-increases with the decrease of cloud fraction not only for reflectance and transmittance but also for column absorption. The overcast case $(\mathrm{N}=1)$ does not produce any visible differences between IPA and MC, at least for the optical cloud model. Inequalities (3a) and (3b) are still valid but the resulting domain-averaged column absorption is essentially the same for both IPA and MC. In our fractal models, increasing gappiness not only increases the clear sky fraction but also increases the number of gaps; hence the number of cloud edges and the effect of horizontal fluxes. With more gaps, more photons are trapped between cloudy pixels, thus accumulating water vapor absorption. As a result, the difference between $\mathrm{T}_{M C}$ and $\mathrm{T}_{\mathrm{TPA}}$ is larger than between $\mathrm{R}_{\mathrm{IPA}}$ and $\mathrm{R}_{\mathrm{MC}}$; hence, $\mathrm{A}_{\mathrm{MC}}>\mathrm{A}_{\mathrm{IPA}}$.

\section{Biases}

It is more convenient to deal not with absolute values of radiative fluxes for different models but with their deviations. We define two types of area-averaged biases.

- "PP" bias determines the changes in radiation fluxes by accounting for the horizontal redistribution of optical depth in cloudy areas (IPA),

$$
\Delta \mathrm{F}_{\text {model }}(\mathrm{PP})=\mathrm{F}_{\text {IPA }}-\mathrm{F}_{\mathrm{PP}}
$$

- "IPA" bias determines the changes in radiation fluxes by accounting for photon horizontal transport (MC),

$$
\Delta \mathrm{F}_{\text {model }}(\text { IPA })=\mathrm{F}_{\mathrm{MC}}-\mathrm{F}_{\text {IPA }}
$$

Figure 4a shows PP column absorption biases for both flat and bumpy models and four solar zenith angles. We see that PP biases are negative and decrease with the increase of solar angle $\theta_{\mathrm{o}}$. This follows directly from Jensen's inequality (for details see Marshak et al. 1997).

Though IPA bias for reflected or transmitted photons can be either positive or negative (Marshak et al. 1997), the IPA bias for column absorption is always positive (Figure 4b), regardless of the cloud model, solar angle and cloud fraction. This means that horizontal fluxes always increase photon path length; hence, 3D radiative effects per se increase column absorption. Next, column absorption for the bumpy cloud model exceeds that of the flat cloud model for all solar angles.

\section{PP Bias}

(a)
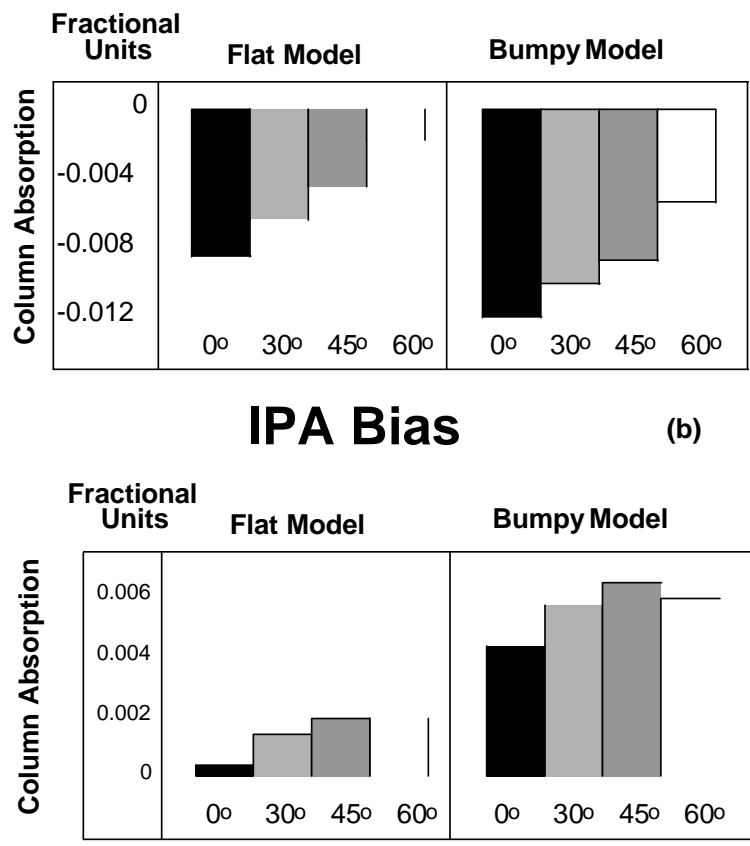

Figure 4. PP and IPA column absorption biases for both cloud models, (a) PP bias, (b) IPA bias.

The maximal IPA bias is reached at about $\theta_{\mathrm{o}}=45^{\circ}$ for fractional absorption. This is true for both cloud models and is consistent with results reported by O'Hirok and Gautier (1996).

Finally, comparing both PP and IPA biases we find that in most cases (for flat model and large cloud cover) the negative PP bias in column absorption exceeds the positive IPA bias. As a result, the total bias, as a sum of both biases, is negative, meaning that the PP absorption is larger than its MC counterpart.

To roughly estimate the 3D effect on total broad-band absorption, we can use the results of Ramaswamy and Friedenreich's (1991) line-by-line shortwave calculations. The ratio of column absorption in the narrow spectral band of $0.9-1.0 \mu \mathrm{m}$ to the total shortwave absorption for different types of plane-parallel clouds is typically $\approx 10$, with an upper limit of $\approx 20$. Thus, if we found that the maximal IPA bias in $0.9-1.0 \mu \mathrm{m}$ band is $0.3 \mathrm{~W} / \mathrm{m}^{2}$, the total enhancement of absorption, which is due to the 3D radiative effects, will likely be around $3 \mathrm{~W} / \mathrm{m}^{2}$, and almost certainly less than $6 \mathrm{~W} / \mathrm{m}^{2}$. This is considerably less than the $15-20 \mathrm{~W} / \mathrm{m}^{2}$ needed to explain "enhanced" absorption. 


\section{Conclusion}

The main results of our analysis for the domain-averaged and narrow band $(0.9-1.0 \mu \mathrm{m})$ averaged fluxes are as follows:

- Redistribution of cloud liquid water decreases column absorption when horizontal fluxes are ignored; in other words, PP column absorption is always larger than its IPA counterpart. This effect is much more pronounced for the bumpy model than for the flat one.

- Horizontal fluxes increase total photon path; MC column absorption is therefore larger than its IPA counterpart. This effect is also more pronounced for the bumpy model than for the flat one; horizontal fluxes enhance IPA column absorption by $0.6 \%$ for the flat model and by up to $2 \%$ for the bumpy model (at about $\theta_{\mathrm{o}} \approx 45^{\circ}$ ).

- The total bias (MC - PP) in absorption is negative for high sun and slightly positive for low sun. In other words, the first-order effect of the redistribution of liquid water (PP bias) has a stronger impact on column absorption than $3 \mathrm{D}$ effects (IPA bias).

The above conclusion on the IPA bias is valid only for the domain-averaged (50-km) absorption. If we average over $10 \mathrm{~km}$ or less, the results become of arbitrary sign and strength, depending on many factors including the distribution of cloud gaps and the solar zenith angle.

In addition, by comparing flat and bumpy models, we find that the IPA estimate of column absorption for the flat model is always larger than its counterpart for the bumpy model. However, the MC column absorption depends rather weakly on the type of model. For the flat model, horizontal fluxes increase reflectance and decrease transmittance; for the bumpy model, they only decrease transmittance and leave reflectance unchanged. As a result, the IPA bumpy model deficit of absorption is compensated for. To summarize, bumpiness enhances horizontal fluxes considerably, leaving column absorption almost unchanged.
Finally, decreasing cloud fraction increases the IPA bias. This is due not only to the increase of the clear sky contribution, but also to the number of gaps; hence, cloud edge-effects in the horizontal fluxes.

Note that the absolute values of the above biases are quite small; they are at most $0.3 \mathrm{~W} / \mathrm{m}^{2}$, which is $\approx 2 \%$ of the column absorption in $0.9-1.0 \mu \mathrm{m}$ spectral band. This is far below the discrepancies between measurements and models reported by Cess et al. (1995) and others. Consequently, we conclude that 3D effects alone do not explain these discrepancies. This argues for alternate mechanisms for absorption enhancement, which may or may not involve clouds.

\section{References}

Cahalan, R. F., and J. B. Snider, 1989: Marine stratocumulus structure during FIRE, Remote Sens. Environ., 28, 95-107.

Cahalan, R. F., W. Ridgway, W. J. Wiscombe, T. L. Bell, and J. B. Snider, 1994: The albedo of fractal stratocumulus clouds. J. Atmos. Sci., 51, 2434-2455.

Cess, R. D., et al. (20 authors), 1995: Absorption of solar radiation by clouds: Observations versus models. Science, 267, 496-499.

O'Hirok, W., and C. Gautier, 1996: The role of threedimensional clouds on atmospheric absorption. J. Atmos. Sci. (submitted).

Marshak, A., A. Davis, W. Wiscombe, and W. Ridgway, 1997: Biases in shortwave column absorption in the presence of fractal clouds. J. Climate. (submitted).

Ramaswamy, V., and S. M. Friedenreich, 1991: Solar radiative line-by-line determination of water vapor absorption and water extinction in inhomogeneous atmospheres. J. Geophys. Res., 96, 9133-9157. 